\title{
APLICAÇÕES DE QCM, EIS E SPR NA INVESTIGAÇÃO DE SUPERFÍCIES E INTERFACES PARA O DESENVOLVIMENTO DE (BIO)SENSORES
}

\author{
Flavio Santos Damos, Renata Kelly Mendes e Lauro Tatsuo Kubota* \\ Instituto de Química, Universidade Estadual de Campinas, CP 6154, 13084-971 Campinas - SP
}

Recebido em 1/9/03; aceito em 13/4/04; publicado na web em 27/07/04

\begin{abstract}
APPLICATIONS OF QCM, EIS AND SPR IN THE INVESTIGATION OF SURFACES AND INTERFACES FOR THE DEVELOPMENT OF (BIO)SENSORS. The use of the quartz crystal microbalance process, electrochemical impedance spectroscopy and surface plasmon resonance for characterizing thin films and monitoring interfaces is presented. The theorical aspects of QCM, EIS and SPR are introduced and the main application areas are outlined. Future prospects of the combined applications of QCM, EIS and SPR methods in the studies of interfacial processes at surfaces are also discussed.
\end{abstract}

Keywords: QCM; EIS; SPR.

\section{INTRODUÇÃO}

Nas últimas décadas, as ciências analíticas têm experimentado um grande avanço relacionado com a capacidade de obtenção de informações químicas de objetos e sistemas. Como resultado, essas informações têm proporcionado um desenvolvimento analítico bem como sua incorporação a outras áreas científicas e técnicas, de forma a resultar na automação, miniaturização e simplificação dos sistemas $^{1}$. Assim sendo, verifica-se o desenvolvimento e o uso apropriado de sistemas de referência, revitalização da análise qualitativa, expansão das fronteiras analíticas clássicas (amostragem, estudos in situ, interdisciplinaridade) e o desenvolvimento de sistemas analíticos como sensores químicos, biossensores, dispositivos bioanalíticos e eletrodos quimicamente modificados. Neste contexto, os sensores químicos e biossensores têm adquirido grande importância devido à possibilidade de aplicação destes sistemas para avaliação de processos sintéticos ou biológicos, bem como o entendimento destes pro$\operatorname{cessos}^{2}$. Sensores químicos são dispositivos que transformam uma informação química, como a variação da concentração de um componente específico de uma amostra em relação à composição total, em um sinal analítico útil. Estes sistemas contêm duas unidades funcionais básicas: um receptor e um transdutor ${ }^{3}$. As aplicações destes no monitoramento e controle ambiental, em agricultura, indústria de alimentos, farmacêutica e clínica têm possibilitado análises com monitoramento contínuo e in vivo em tempo real ${ }^{4}$.

O desenvolvimento de dispositivos sensores tem como premissa básica a avaliação de processos interfaciais e superficiais, uma vez que estes processos são de fundamental importância no entendimento dos mecanismos de interação entre o sistema de reconhecimento e o analito ${ }^{5,6}$. Além disso, o estudo de processos interfaciais e superficiais é de grande relevância para a escolha do método de imobilização de espécies sobre a superfície de eletrodos e a avaliação de problemas associados aos métodos de imobilização. Assim, o estudo destes processos pode auxiliar na escolha do melhor meio de imobilização a ser usado e indicar as principais características (vantagens e desvantagens) associadas a cada método de imobilização ${ }^{7-12}$.

Desta forma, há de se ressaltar a relevante importância que tem adquirido as técnicas capazes de avaliar fenômenos em superfícies e interfaces, incluindo métodos espectroscópicos, microscópicos e/ou

*e-mail: kubota@iqm.unicamp.br acústicos como espectroscopia de reflexão total atenuada ${ }^{13}$, elipsometria ${ }^{13}$, ressonância de plásmons de superfície (SPR - "surface plasmon resonance") ${ }^{14}$, espectroscopia Raman ${ }^{15}$, espectroscopia de impedância eletroquímica (EIS - "electrochemical impedance spectroscopy") $)^{16}$, microscopia de tunelamento de elétrons ${ }^{17}$, microscopia de força atômica ${ }^{18}$, microbalança de cristal de quartzo (QCM - "quartz cristal microbalance") $)^{19}$ e outros. Contudo, limitações no uso de técnicas como elipsometria, devido a sua menor conveniência para monitoramento de dinâmica de eventos ${ }^{20}$, e reflexão total atenuada pela baixa sensibilidade para avaliação de processos superficiais $^{21}$, têm direcionado grande atenção para a QCM ${ }^{22}$, EIS $^{23}$ e SPR ${ }^{24}$. A espectroscopia de impedância eletroquímica tem adquirido grande importância, pois possibilita a obtenção de grande número de informações a partir de um único experimento ${ }^{23}$, bem como a obtenção de informações complementares às obtidas por SPR e QCM. Assim sendo, neste trabalho são discutidos os princípios básicos e aplicações individuais e combinadas das técnicas QCM, EIS e SPR com o propósito de apresentar a importância destas na investigação de processos superficiais e interfaciais, ressaltando suas complementaridades.

\section{PRINCÍPIOS BÁSICOS DE QCM, EIS E SPR}

\section{Microbalança de cristal de quartzo}

Consiste, basicamente, em um disco confeccionado em cristal piezelétrico de quartzo revestido nas faces superior e/ou posterior por um depósito metálico, como mostra a Figura 1a. Este, por sua vez, é acoplado a um circuito oscilador responsável pela aplicação do campo elétrico alternado ao cristal, cujo propósito é provocar uma oscilação no centro do cristal piezelétrico ${ }^{25}$. Diretamente ligado ao circuito oscilador aparece um contador de freqüência (freqüencímetro) responsável pelo monitoramento das variações na freqüência de oscilação do cristal e um computador para obtenção e tratamento dos dados, conforme apresentado na Figura 1b. À medida que o potencial elétrico alternado é aplicado, promovendo um movimento vibracional no cristal, estabelece-se uma onda acústica transversa que se propaga através do cristal. Como resultado ocorre um deslocamento dos átomos do cristal paralelo à sua superfície. Desta forma, se um material é depositado sobre a superfície do cristal ocorre uma redução no movimento de oscilação, resultando em diminuição 
(a)

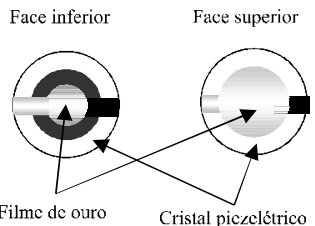

(c)

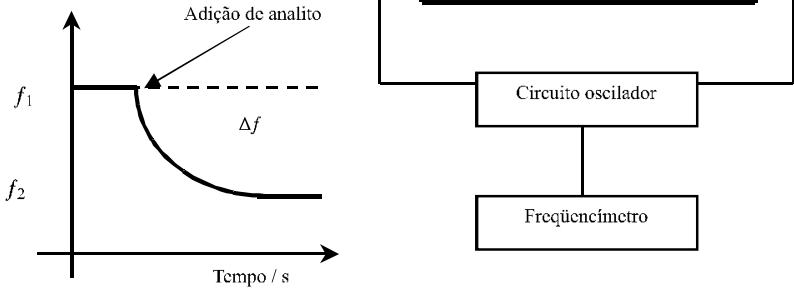

Figura 1. (a) Desenho esquemático da vista superior e inferior de um cristal de quartzo com depósitos metálicos em ambos os lados; (b) esquema ilustrativo de uma célula de fluxo para operação de cristais piezelétricos em meios líquidos, incluindo o sistema oscilador e o frequencímetro; (c) massograma esquemático representativo da variação da freqüencia de um cristal de quartzo em função do tempo. ( $\left(_{1}\right.$, ) freqüência inicial do cristal; $\left(\begin{array}{l}i_{2} \\ i_{2}\end{array}\right)$ freqüência após adição do analito; (D $\left.{ }_{1}\right)$ variação da freqüência do sistema

da frequiência de ressonância do mesmo. Conseqüentemente, mediante um monitoramento contínuo da freqüência do cristal é possível se investigar os fenômenos interfaciais e superficiais (Figura 1c).

Como apresentado nos trabalhos pioneiros de Sauerbrey ${ }^{26}$, a freqüência da oscilação ressonante é função da massa do cristal, de forma que mudanças na massa do sistema, devido à deposição de espécies sobre a superfície do cristal, podem ser monitoradas pelo acompanhamento das mudanças na freqüência deste (Figura 1c) conforme descrito pela equação ${ }^{26}$

z score entre laboratórios $=\frac{\mathrm{S}-\mathrm{md}(\mathrm{S})}{\mathrm{IQN}(\mathrm{S})}$

onde, $r_{q}$ é a densidade do quartzo, $m_{q}$ - módulo de cisalhamento do cristal, $f_{\mathrm{o}}$ - freqüêencia fundamental do cristal e $A$ - área geométrica piezeletricamente ativa do cristal que, por sua vez, é definida pela projeção dos filmes metálicos depositados sobre o cristal, $\Delta m$ e $\Delta f$ correspondem às variações na massa e freqüência do sistema.

Neste contexto, os fundamentos teóricos desenvolvidos por Sauerbre ${ }^{26}$ foram posteriormente aplicados por King ${ }^{27}$ no desenvolvimento do primeiro dispositivo detector de sorção piezelétrico para hidrocarbonetos como o-xileno, etil-benzeno, n-octano, tolueno, nheptano e n-pentano. $\mathrm{O}$ sistema consistia no emprego de um cristal piezelétrico de quartzo (CPQ) modificado, como dispositivo detector, e outro idêntico não modificado usado como dispositivo de referência, conduzindo a uma nova classe de dispositivos detectores rápidos e seletivos.

Com os avanços inicialmente obtidos por King ${ }^{27}$ surgiram diferentes configurações e mecanismos de interação entre a onda acústica e a matéria, resultando em inúmeras aplicações em sistemas que utilizam os CPQ como leitores de massa, dispositivos eletroacústicos e por efeito elástico. Desta forma, as diferenças presentes nestes sistemas originaram um grande número de dispositivos fundamentados em materiais piezelétricos, como osciladores de onda acústica estrutural (BAW - "Bulk Acoustic Wave Resonators"), osciladores de onda acústica de superfície (SAW - "Surface Acoustic Wave Resonators"), osciladores de onda acústica em membranas flexíveis (FPW - "Flexural Plate Wave"), osciladores de onda acústica em fibra (FAW - "Fibre Acoustic Wave"), osciladores de onda acústica em tubo (TAW - "Tube Acoustic Wave") e dispositivos de onda acústica transversa (TWD - "Transverse Wave Devices").

As aplicações iniciais da QCM a ambientes líquidos não lograram êxito, uma vez que o cristal de quartzo cessava o movimento de oscilação quando imerso em solução e, desta forma, as análises eram conduzidas mediante a conversão da amostra à fase gasosa ou através de processos exaustivos de imersão do cristal na solução ${ }^{28-30}$.

A primeira aplicação, com sucesso, de um dispositivo acústico como sensor em fase líquida foi conduzida por Konash e Bastiaans ${ }^{31}$, através do emprego de cristais piezoelétricos de quartzo como detectores de massa para cromatografia líquida. O desenvolvimento deste sistema possibilitou a superação de dois problemas principais associados ao uso dos CPQ em ambientes líquidos, que são: oscilação estável do cristal e a eliminação dos efeitos provenientes da viscosidade e densidade do líquido.

Para a aplicação da QCM a sistemas líquidos há de se considerar fatores como a densidade $(\rho)$ e a viscosidade $(\eta)$ do líquido, uma vez que o acoplamento da superfície do cristal a um líquido muda drasticamente a freqüência de oscilação deste. Esta mudança é ocasionada pelo movimento de cisalhamento do cristal, que gera um movimento no líquido próximo à interface (fluxo plano-laminar no líquido) causando uma diminuição na freqüência de oscilação do cristal proporcional a $(\rho \eta)^{1 / 2} 28$, conforme pode ser verificado na expressão

$\Delta f=f_{o}^{32} \sqrt{\frac{\rho \eta}{\pi \rho_{q} \mu_{q}}}$

onde $r_{q}$ é a densidade do quartzo, $\mathrm{m}_{q}$ - módulo de cisalhamento do cristal, $f_{o}$ - frequiência fundamental do cristal, p representa a constante 3,1416, r a densidade e $\mathrm{h}$ a viscosidade do líquido.

Com o propósito de otimizar a aplicação de CPQ a ambientes líquidos, inúmeros trabalhos foram desenvolvidos a partir dos estudos de Nomura e Okuhara ${ }^{32}$. Com estes estudos foi desenvolvida uma equação empírica que relaciona a freqüência de ressonância do cristal, a viscosidade e densidade da solução para líquidos não condutores.

Após os trabalhos conduzidos por Nomura e Okuhara ${ }^{32}$, outros grupos de pesquisa comprovaram que, além da viscosidade e densidade do líquido, parâmetros como estrutura de interface sólido/solução ${ }^{33}$, condutividade, polaridade e temperatura ${ }^{34-36}$, viscosidade interfacial e características hidrofílicas e hidrofóbicas da superfície do cristal $^{37-41}$, uniformidade do filme sobre o cristal ${ }^{36}$ e extensão da área do cristal em contato com a solução ${ }^{42}$ são de grande relevância para a aplicação da QCM a ambientes líquidos.

\section{Espectroscopia de impedância eletroquímica}

Envolve a aplicação de uma perturbação de potencial ou de corrente no sistema sob investigação. A perturbação do sistema é feita mediante a aplicação de um potencial contínuo (potencial central aplicado) sobre a qual é superimposta uma variação senoidal de potencial com pequena amplitude. Este método de aplicação do potencial possibilita que o sistema seja perturbado empregando poucos milivolts, de forma a tornar possível a investigação de fenômenos eletroquímicos próximos ao estado de equilíbrio. Além disto, é possível perturbar o sistema usando diferentes valores de freqüência, pois a onda de potencial é senoidal. Uma vez que a perturbação no sistema sob investigação é de pequena amplitude é possível empregar a técnica para a análise de etapas de um mecanismo reacional ${ }^{43,44}$.

Na EIS surge uma corrente de natureza senoidal como resultado da aplicação de um potencial senoidal ao sistema. Mediante um monitoramento das relações entre o potencial aplicado e a corrente são obtidas a impedância do sistema e o ângulo de fase (defasagem da corrente em relação ao potencial aplicado). 
O conceito de impedância, originalmente introduzido para descrever a resposta de sistemas compostos por capacitâncias, resistências e indutâncias, estendeu-se aos sistemas eletroquímicos, uma vez que inúmeros processos podem contribuir para a relação entre a corrente e o potencial do sistema. Assim, a partir das medidas da impedância e ângulo de fase é possível avaliar processos como transporte de carga (incluindo estimativa de velocidade de transferência), condutividade de filmes, capacitância redox e de dupla camada, coeficientes de difusão de portadores de carga ${ }^{23}$, entre outros.

A obtenção de informações a partir dos dados de impedância eletroquímica pode ser conduzida mediante a utilização de diferentes modelos de medida, como circuitos equivalentes ou modelos matemáticos. A aplicação de circuitos equivalentes tem como fundamento as similaridades entre o comportamento da célula eletroquímica (Figura 2a) e um circuito elétrico de resistores, capacitores e indutores. Uma equivalência típica entre um circuito equivalente e um sistema eletroquímico é apresentada na Figura 2b. O comportamento similar da dupla camada elétrica a um capacitor de placas paralelas (modelo de Helmholtz ${ }^{44}$ ) e a resistência à transferência de carga na interface eletrodo/solução a um resistor possibilita uma representação da interface por uma associação em paralelo entre um resistor $\left(R_{c c}\right)$ e um capacitor $\left(\mathrm{C}_{\mathrm{d}}\right)$, devido à contribuição dos processos faradáicos $\mathrm{e}$ capacitivos. Uma vez que a corrente que passa na interface eletrodo/ solução é conduzida pelos íons em solução, o efeito resistivo na solução sobre a migração dos íons é representado por uma resistência $\mathrm{R}_{\mathrm{W}}$. Por outro lado, a introdução de elementos capacitivos em um circuito promove uma defasagem entre a corrente e o potencial. Desta forma, uma representação comum para a impedância em sistemas compostos por resistores e capacitores é através de um diagrama de fasores no qual a impedância apresenta uma componente real (resistiva) e imaginária (capacitiva). Para o circuito equivalente apresentado (Figura 2b) as componentes real (Z') e imaginária (Z”) da impedância do sistema são ${ }^{44}$
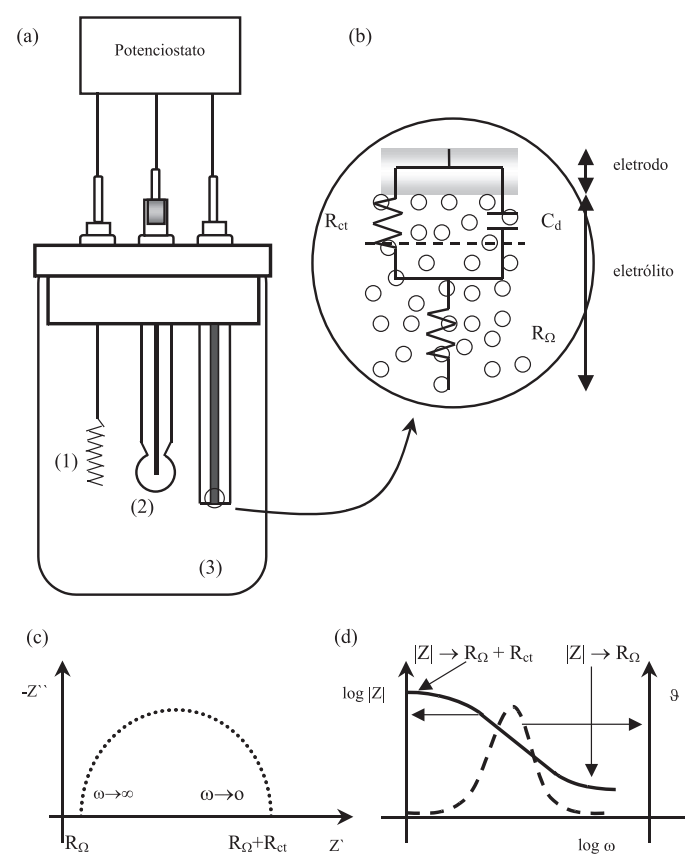

Figura 2. (a) Célula eletroanalítica típica de três eletrodos para uso em EIS: (1) eletrodo auxiliar, (2) eletrodo de referência e (3) eletrodo de trabalho; (b) diagrama esquemático de um circuito Randles superimposto à interface eletrodoleletrólito; (c) diagrama de impedância (diagrama de Nyquist) referente ao circuito equivalente apresentado na figura; (d) diagrama de Bode correspondente ao diagram de Nyquist apresentado
$Z^{\prime}=R_{\Omega}+\frac{R_{c t}}{1+\omega^{2} C_{c t}^{2} R_{c t}^{2}}$ e $Z^{\prime \prime}=\frac{\omega C_{2} R_{c t}^{2}}{1+\omega^{2} C_{c t}^{2} R_{c t}^{2}}$

onde, $R_{\Omega}$ - resistência da solução, $R_{c t}$ - resistência à transferência de carga, $\omega$ - frequiência angular e $C_{d}$ - capacitância da dupla camada elétrica.

Conforme pode ser observado nas expressões (3) na região de altas freqüências a componente real da impedância tende a $R_{\Omega}$, enquanto que nas regiões de baixa freqüência tende a $R_{\Omega}+R_{c t}$. Ao avaliar este comportamento é possível inferir como obter informações sobre a resistência da solução na região de altas freqüências e na região de baixas freqüências sobre os processos de eletrodo (resistência à transferência de carga).

Por outro lado, eliminando a freqüência nas expressões anteriores obtém-se a relação entre a componente imaginária e a componente real da impedância, conforme apresentado a seguir

$\left(Z^{\prime}-R_{\Omega}-\frac{R_{c t}}{2}\right)^{2}+Z^{\prime \prime 2}=\left(\frac{R_{c t}}{2}\right)^{2}$

Pode ser verificado na expressão anterior que um gráfico de $Z^{\prime \prime}$ em função de $Z^{\prime}$ resulta num comportamento circular (Figura 2a) com raio de $R_{c t} / 2$ e centro em $Z^{\prime}=R_{\Omega}+R_{c t} / 2$ e $Z^{\prime \prime}=0$. Neste contexto, a representação gráfica $Z^{\prime \prime}$ (componente imaginária da impedância) vs $Z^{\prime}$ (componente real da impedância), também denominado diagrama de Nyquist, Argand, Sluyters ou gráfico de Cole-Cole (Figura 2c), pode fornecer informações sobre as possíveis naturezas dos elementos que constituem a impedância total do sistema ${ }^{45,46}$.

Adicionalmente, representações de $\log |Z|(|Z|$ - módulo da impedância) e $\varphi$ (ângulo de fase) vs log $\omega$ ( $\omega$ - freqüência), chamadas de curvas de Bode (Figura 2d), são de grande importância para a interpretação de dados provenientes da EIS, pois as informações obtidas a partir destes gráficos podem ser complementares às obtidas no diagrama de Nyquist. Conforme pode ser observado na Figura 2d, as alterações no módulo da impedância indicam os efeitos da resistência à transferência de carga no sistema. Por outro lado, à medida que a freqüência do sistema é aumentada observam-se alterações no ângulo de fase entre o potencial aplicado e a corrente resultante. Esta alteração no ângulo de fase possibilita a obtenção de informações sobre a componente capacitiva do sistema, uma vez que as componentes capacitivas introduzem um comportamento co-senoidal na corrente $e^{47}$.

Desta forma, os efeitos da transferência de carga $\left(\mathrm{R}_{\mathrm{tc}}\right)$, da dupla camada elétrica $\left(\mathrm{C}_{\mathrm{d}}\right)$, bem como os efeitos da solução sobre a migração de íons $\left(\mathrm{R}_{\mathrm{w}}\right)$, podem ser obtidos mediante a avaliação de diagramas de Nyquist e curvas de Bode.

Adicionalmente, nos sistemas que apresentam efeitos significativos da impedância à transferência de massa $\left(\mathrm{Z}_{\mathrm{tm}}\right)$ a introdução de um elemento denominado "impedância Warburg" é feita com o propósito de simular as características do sistema experimental, tanto em processos que sofrem difusão linear, esférica ou sob convecção forçada. Neste contexto, um grande conjunto de fatores pode influenciar a impedância do sistema, como processos faradaicos, adsorção de espécies eletroativas e não-eletroativas, reações no eletrodo envolvendo a formação de intermediários estáveis e heterogeneidade de superfíci$\mathrm{es}^{43}$, de forma a proporcionar à EIS um amplo campo de aplicação.

\section{Ressonância de plásmons de superfície}

Onda de Plásmons de Superfície (OPS) são ondas eletromagnéticas longitudinais, que se propagam na interface entre um metal e um dielétrico. Estas ondas têm sido exploradas pela técnica SPR pois podem receber energia de uma radiação que se propaga pela interface. Contudo, para que a OPS entre em ressonância com a radi- 
ação incidente é necessário que ambas tenham vetores de onda de igual magnitude. Uma vez que o vetor de propagação da OPS é superior ao da onda incidente, não ocorre excitação da OPS mediante o contato direto destas ondas eletromagnéticas. Portanto, foram desenvolvidos sistemas ópticos para elevar o momento da onda incidente, de forma que a OPS possa receber energia da onda incidente através de uma transferência ressonante de energia (Figura 3a). Neste sentido, o fenômeno da reflexão total atenuada bem como a difração sobre grades de difração têm sido explorados ${ }^{48}$ mediante o emprego da configuração Kretschmann ${ }^{49}$ ou através de guias de onda ópticos ${ }^{49}$.

Os sistemas SPR que operam mediante o emprego da configuração Kretschmann são os de maior uso dentre as configurações, pois geralmente apresentam maior sensibilidade e resolução em relação aos dispositivos que operam por grades de difração $0^{49,50}$. Nestes sistemas, durante a reflexão interna total ocorre a propagação de uma fração da onda incidente na interface, de forma a penetrar no meio de menor densidade óptica dando origem a um campo eletromagnético evanescente (Figura 3a). Ao se estender até o ambiente esta fração da radiação incidente acopla-se aos elétrons livres oscilantes do metal, de forma que alterações nas proximidades da interface metal/ ambiente promovam uma alteração nas condições de ressonância do sistema. Como resultado, ocorre um deslocamento no ângulo SPR (Figura 3b). Conforme pode ser verificado na expressão a seguir, a componente paralela do fóton de luz incidente tem o vetor onda $\left(k_{\mathrm{x}}\right)$ relacionado com o ângulo de incidência da luz

$k_{x}=\frac{\omega}{c} \sqrt{\varepsilon_{p}} \operatorname{sen} \Theta$

onde $\Theta$ é o ângulo de incidência da luz com a superfície do metal, $\varepsilon_{p}$ é a constante dielétrica do prisma, $\omega$ é a freqüência angular da luz incidente e $c$, a velocidade da luz. Adicionalmente, a constante de propagação da OPS $\left(k_{O P S}\right)$ numa interface metal/dielétrico é expressa por:

$k_{O P S}=\frac{\omega}{c} \sqrt{\frac{\varepsilon_{m} \varepsilon_{a}}{\left(\varepsilon_{a}+\varepsilon_{m}\right)}}$

onde $\omega$ é a frequiência angular da onda, $c$ é a velocidade da luz, $\varepsilon_{p}$ é a constante dielétrica da amostra (espécies que interagem com a superfície) e $\varepsilon_{m}$ é a constante dielétrica do metal. Assim, é possível utilizar o ângulo da luz incidente como um parâmetro de controle do fenômeno de OPS, mediante um monitoramento da refletância vs ângulo de inci-

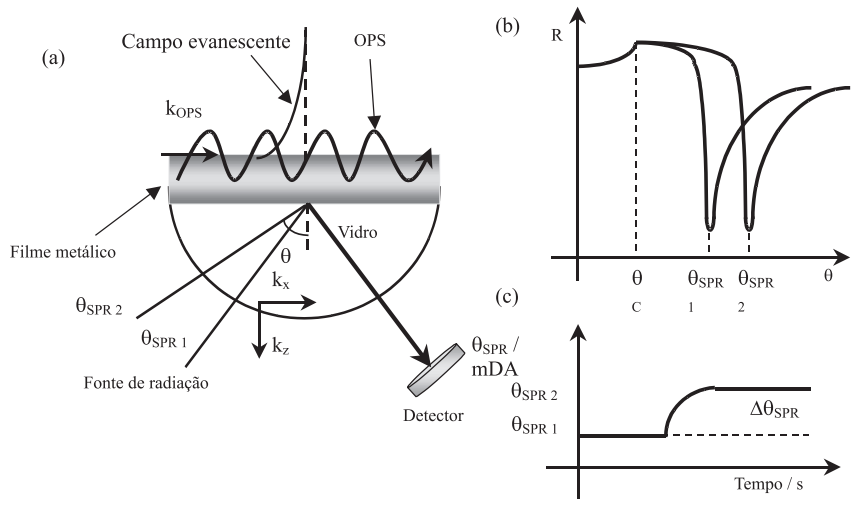

Figura 3. (a) Representação esquemática da configuração de Kretchmann para SPR. O dielétrico em contato com o metal possibilita a transferência ressonante de energia da onda incidente para a OPS; (b) Curvas de refletância na ausência $\left(\theta_{S P R I}\right)$ e presença $\left(\theta_{S P R 2}\right)$ de espécies na superfície do filme metálico; (c) Sensorgrama esquemático representativo da relação entre o ângulo $\operatorname{SPR}\left(\theta_{S P R}\right)$ e tempo durante a interação de espécies com a superfície do filme metálico dência de luz (Figura 3b). No instante em que a componente paralela do fóton de luz incidente é equivalente à constante de propagação da OPS, ou seja, $k_{x}=k_{\text {OPS }}$ (Equações 5 e 6 ) deve ser verificada uma queda na refletância, devido à formação da onda evanescente, que se propaga através da superfície metálica e interage com o meio externo, como pode ser verificado através das expressões 7 e 8.

$\frac{\omega}{c} \sqrt{\varepsilon_{p}} \operatorname{sen} \Theta=\frac{\omega}{c} \sqrt{\frac{\varepsilon_{m} \varepsilon_{a}}{\left(\varepsilon_{m}+\varepsilon_{a}\right)}}$

$\Theta=\operatorname{arcsen}\left(\sqrt{\left(\frac{\varepsilon_{m} \varepsilon_{a}}{\left(\varepsilon_{m}+\varepsilon_{a}\right)} \times \frac{1}{\varepsilon_{p}}\right)}\right)$

Nesta última expressão pode-se verificar que as propriedades ópticas do sistema, como as constantes dielétricas do metal, do prisma e da matriz, provocam mudanças no ângulo de ressonância, tornando possível aplicar o fenômeno da SPR ao monitoramento de alterações na superfície do sensor, mediante um acompanhamento do ângulo de ressonância com o tempo (Figura 3c). Assim, mediante o monitoramento do índice de refração nas proximidades da superfície do disco sensor é possível aplicar a SPR para se obter informações sobre velocidade e extensão da adsorção, possibilitando a determinação de propriedades dielétricas, cinética de associação e dissociação, bem como constantes de afinidades em interações específicas ${ }^{24}$.

Nos sistemas que operam por grades de difração, a onda ao incidir sobre a grade de difração (componente óptico que modula periodicamente a fase ou amplitude da onda incidente) é "dividida" em uma série de feixes, de forma que sua componente do vetor onda paralela à superfície sofre alterações dando origem aos vetores onda da luz difratada. Desta forma, estas alterações na radiação possibilitam uma transferência de energia entre a onda incidente e a OPS, semelhantemente aos sistemas que operam mediante a reflexão total atenuada.

A partir da teoria individual de cada técnica é possível avaliar seus campos aplicativos, bem como estabelecer suas potencialidades e limitações. Neste contexto, mediante uma verificação da teoria associada com a QCM e SPR observa-se que as ondas acústicas e as ondas de plásmons de superfície não são fenômenos onde ocorre perda de energia por radiação, mas por ondas evanescentes. Assim sendo, é possível estabelecer limites teóricos para a extensão da penetração destes campos evanescentes, conforme pode ser verificado através das expressões a seguir ${ }^{22}$

$\alpha_{\mathrm{MCQ}}=(2 \eta / \omega \rho)^{1 / 2}$

$\alpha_{\mathrm{SPR}}=1 / 2 \pi(\varepsilon)^{1 / 2}$

onde $\alpha_{\mathrm{MCQ}}$ e $\alpha_{\mathrm{SPR}}$ são a extensão de penetração das ondas evanescentes geradas na QCM e na SPR. $\eta$ e $\rho$ são, respectivamente, a viscosidade e a densidade do líquido onde está imerso o cristal, $\omega$ é a frequiência de excitação angular do cristal, $\lambda$ é o comprimento da onda incidente, $\pi$ é a constante 3,1416 e $\varepsilon$ a constante dielétrica do líquido de imersão. Por outro lado, uma vez que as medidas de impedância eletroquímica têm por fundamento a perturbação do sistema mediante aplicação de um potencial ou corrente ao sistema, a EIS não sofre das mesmas limitações impostas à SPR e QCM.

\section{PROCESSOS INTERFACIAIS, SUPERFICIAIS E APLICAÇÕES ANALÍTICAS DA QCM, EIS E SPR}

O desenvolvimento da teoria associada à aplicação da microbalança de cristal de quartzo a ambientes líquidos possibilitou seu uso em caracterizações de sistemas biomoleculares, tendo como 
propósitos o desenvolvimento de sistemas sensores, bem como a investigação de processos interfaciais para o estudo destes sistemas ${ }^{51,52}$. Okahata e colaboradores ${ }^{53}$ realizaram medidas cinéticas da hibridização de DNA sobre cristais piezelétricos de quartzo com $27 \mathrm{MHz}$ de freqüência fundamental, modificados com oligonucleotídeos complementares através de pontes avidina-biotina.

Com estas bases tem surgido um grande número de trabalhos investigativos de hibridização entre oligonucleotídeos e possíveis agentes danosos ao ácido desoxirribonucléico ${ }^{54}$. Cosnier e colaboradores ${ }^{55}$ empregaram a microbalança de cristal de quartzo para realizar uma investigação dos processos microgravimétricos de eletrogeração de filmes de polipirrol-biotina, com o propósito de produzir matrizes com alto grau de reprodutibilidade referente ao processo de eletrodeposição, bem como deposição de macromoléculas biológicas, como glicose oxidase.

A aplicabilidade dos cristais piezelétricos de quartzo estende-se às investigações de processos de transferência de carga e massa em metalopolímeros, uma vez que estes fenômenos influenciam diretamente parâmetros, como o coeficiente de transporte de carga aparente, que são de fundamental importância para a interpretação dos fenômenos de transporte de carga e massa em filmes poliméricos ${ }^{56,57}$. Além disso, o uso da microbalança de cristal de quartzo possibilita o estudo separado dos fenômenos de transferência de carga e de massa, de forma a contribuir, fundamentalmente, para a investigação de fenômenos interfaciais ${ }^{58}$.

Neste sentido, a aplicação da microbalança de cristal de quartzo proporciona um estudo isolado de fenômenos interfaciais, de forma a possibilitar uma distinção entre os diferentes tipos de reações interfaciais, permitindo uma classificação dos possíveis tipos de reações. Fletcher e colaboradores ${ }^{58}$ utilizaram a microbalança de cristal de quartzo para gerar massogramas que, juntamente com dados voltamétricos, gerou uma classificação das reações interfaciais. Es- tes estudos conduziram à classificação das reações interfaciais em, basicamente, cinco tipos: reações onde ocorrem processos faradaicos e estes estão associados a mudanças na massa, como eletrodeposição, eletrodissolução e reações de intercalação; reações onde ocorrem processos faradaicos que não estão associados a mudanças na massa, como reações de evolução de gases; processos não-faradaicos que não estão associados a mudanças de massa, como os processos capacitivos; processos não-faradaicos associados com variações de massa, como processos de adsorção específica de íons perclorato sobre superfície de ouro e processos em que ocorrem mudanças na massa, porém não estão associados a processos não-faradaicos ou faradaicos. Alguns exemplos de estudos de processos interfaciais relacionados com o desenvolvimento de sensores piezelétricos estão apresentados, resumidamente, na Tabela 1.

Além da aplicação como ferramenta para a investigação de fenômenos de interação com o propósito de desenvolvimento de sistemas biossensíveis, os cristais piezelétricos de quartzo têm sido intensamente aplicados em estudos de cinética de adsorção nas mais diversas matrizes. Neste sentido, têm sido alvo de constantes investigações a cinética de adsorção de proteínas ${ }^{59}$, alcanotióis ${ }^{60}$, espécies surfactantes como dodecilsulfato de sódio ${ }^{61}$, metais como cobre ${ }^{62}$, entre outros. Estes estudos são proporcionados pela capacidade da técnica em conduzir medidas simultâneas de mudanças na freqüência e medidas de dissipação de energia do cristal piezelétrico de quartzo, durante o processo de adsorção ${ }^{63}$.

Mediante um monitoramento da freqüência de ressonância de cristais piezelétricos é possível avaliar as mudanças na massa e, conseqüientemente, estudar diferentes processos interfaciais em filmes finos, possibilitando a avaliação de transporte de espécies, cinética de adsorção e crescimento.

Neste contexto de investigação de fenômenos de superfície e interfaces, a espectroscopia de impedância eletroquímica tem sido am-

Tabela 1. Exemplos de aplicação da microbalança de cristal de quartzo em investigação de processos interfaciais e superficiais

\begin{tabular}{|c|c|c|}
\hline Aplicação & Propósito & Ref \\
\hline Filmes orgânicos & $\begin{array}{l}\text { - uso de um dispositivo BAW para avaliação dos efeitos da espessura do filme e } \\
\text { solventes no processo de "cura" do filme } \\
\text { - estudo dos parâmetros associados com a adsorção por QCM, bem como } \\
\text { identificaçãa e divisão entre a adsorção física e química durante o estágio } \\
\text { de formação do filme }\end{array}$ & 93 \\
\hline Filmes de Langmuir-Blodgett & $\begin{array}{l}\text { - monitoramento da espessura de filmes de 1,4,8,11,15,18,22,25-octabutoxi- } \\
\text { ftalocianina de cobre (II) } \\
\text { - estudo de adsorção e desorção de lipossomas em soluções e emulsões }\end{array}$ & $\begin{array}{l}94 \\
95\end{array}$ \\
\hline Monocamadas auto-organizadas & $\begin{array}{l}\text { - estudo da interação de globotriaosilceramida (Gb3) com as toxinas "shiga" } \\
1 \text { e } 2 \\
\text { - estudo da cinética de formação de butanotióis sobre ouro }\end{array}$ & $\begin{array}{l}96 \\
97\end{array}$ \\
\hline Filmes protéicos & $\begin{array}{l}\text { - cinética de adsorção e propriedades viscoelásticas de Proteína A (proveniente } \\
\text { de Staphylococcus aureus), albumina de soro bovino, Imunoglobulina G } \\
\text { e fibronectina } \\
\text { - estudo da interação (em tempo real) e cálculo das constantes de associação, } \\
\text { dissociação e de equilíbrio de cloreto de berberina com albumina de soro bovino }\end{array}$ & 98 \\
\hline Sistemas supramoleculares & $\begin{array}{l}\text { - estudo de interação enantioseletiva de filmes de calix[4]arenos com (R) } \\
\text { metil-lactato e (S) metil-lactato } \\
\text { - cinética de adsorção e desorção de vapores de tolueno e clorofórmio } \\
\text { sobre filmes de t-butil-calix[6]areno }\end{array}$ & 103 \\
\hline
\end{tabular}


plamente aplicada, com propósito complementar, aos mais variados setores da eletroquímica, como cinética de eletrodo ${ }^{64-66}$, estudos de dupla camada $^{67}$, processos em baterias ${ }^{68}$, investigação sobre processos de corrosã $0^{69,70}$, eletroquímica em estado sólido ${ }^{71}$ e bioeletroquímica ${ }^{72}$, fundamentalmente para investigação de processos em membranas.

Mediante o emprego de EIS, Heiduschka e colaboradores ${ }^{73}$ desenvolveram monacamadas auto-organizadas mistas de $\omega$ undecanotiol sobre ouro modificadas com fragmentos de antígeno viral (epitopos), com o propósito de reduzir a necessidade de métodos de separação entre o antígeno e o anticorpo, minimizando o dano ao receptor. Uma vez confeccionadas as monocamadas, as medidas de impedância foram utilizadas para permitir o cálculo da componente capacitiva do sistema, para o monitoramento contínuo de interações entre o antígeno e o anticorpo. Neste contexto, Farace e colaboradores $^{74}$ aplicaram a EIS para desenvolvimento de um sistema sensor para dispositivos que operam por afinidade, como interações antígeno - anticorpo e interações entre cadeias complementares de oligonucleotídeos. Ouerghi e colaboradores ${ }^{75}$ aplicaram a EIS num sistema contendo poli-2-ciano-etilpirrol modificado com anticorpo policlonal de coelho como sensor para o antígeno policlonal de coelho. A EIS foi aplicada na investigação do aumento da resistência do polímero, associado com o incremento da resistência à transferência de carga, bem como para avaliar a redução da impedância difusional do sistema, pois possibilita obter informações relevantes sobre as condições de operação otimizadas do eletrodo de trabalho.

Com o propósito de estabelecer as melhores condições de construção dos filmes ${ }^{76}$, tem sido, também, relatada a aplicação da EIS na estimativa do grau de cobertura de superfícies por depósitos de calix[4]areno. A aplicação da EIS tem indicado a uniformidade de filmes em multicamadas ${ }^{77,78}$, de forma a estabelecer as melhores condições para construção de filmes com resistência e compactos, porém com alto grau de sensibilidade ao analito.

A aplicação da EIS na investigação de mecanismos de transferência de carga em filmes, como polipirrol modificados com avidina ou anticorpos tem sido realizada mediante avaliação do comportamento da impedância do sistema em função da freqüência de excitação. Estas investigações têm o propósito de indicar as relações entre as alterações no polímero e as interações resultantes da bioafinidade entre o receptor e o analito ${ }^{79}$. Neste contexto, Kasen ${ }^{80}$ aplicou a EIS para avaliar processos de difusão e cinéticos concomitantes em eletrodos de carbono vítreo modificados com filmes de ferro-hexacianorutenato (II), mediante a avaliação de constantes cinéticas de diferentes parâmetros relacionados com a difusão, transferência de carga e capacitância de dupla camada.

As aplicações analíticas da EIS têm se estendido às investigações de hidridização de oligômeros de DNA, mediante o acompanhamento da impedância total do sistema. Estas investigações são possíveis pois a impedância total do sistema está associada ao aumento da componente capacitiva do sistema. Além disso, a componente capacitiva do sistema é resultante de mudanças na densidade e mobilidade de íons associados com a reação de hibridização ${ }^{81}$. Vetterl e colaboradores ${ }^{82}$, com o propósito de avaliar diferenças entre a conformação duplo-helíptica e a conformação simples, bem como a cinética de adsorção e mobilidade de segmentos de polinucleotídeos adsorvidos, avaliaram a capacitância diferencial de dupla camada em eletrodos contendo DNA adsorvido.

A aplicação da EIS tem se estendido à investigação de propriedades condutoras de polímeros como poliacetileno, polipirrol, politiofeno, polianilina, entre outros. Munichandraiah e Prasad ${ }^{83}$ avaliaram a capacidade catalítica de eletrodos de platina modificados com o polímero polianilina na oxidação de $\mathrm{Fe}^{2+}$ e hidroquinona. Através de uma estimativa da eficiência catalítica do sistema, resultante da corrente de equilíbrio e da constante de velocidade intrínseca, estes estudos conduziram a aplicações otimizadas destes polímeros em sistemas de proteção à corrosão, baterias, catalisadores em reações de oxidação, entre outras ${ }^{84}$. A Tabela 2 apresenta, resumidamente, algumas aplicações da EIS relacionadas com o desenvolvimento de biossensores impedimétricos.

Tabela 2. Exemplos de aplicação da espectroscopia de impedância eletroquímica na investigação de processos interfaciais e superficiais

Aplicação Propósito Pef.

Filmes orgânicos

Filmes de Langmuir-Blodgett

Monocamadas auto-organizadas

Filmes protéicos

Polímeros condutores

Sistemas supramoleculares
- estimar as constantes de equilíbrio ácido-base $(\mathrm{pK})$ para monocamadas de ácido mercapto propiônico, ácido mercapto hexadecanóico e mercaptododecilamina

- investigar processos de transferência de elétrons, incluindo resistência à transferência de carga e capacitância faradaica em filmes poliméricos de poli(N-dodecilacrilamida)-co-(4(acrilometil)4'-metil-2,2'-bipiridina)bis(2,2'-bipiridina) diperclorato de rutênio

- estudar processos de transferência de carga e massa em filmes híbridos de dimetildioctadecilamônio e azul da Prússia

- estudar a cinética de adsorção e grau de cobertura para SAMs de octanotiol, decanotiol, dodecanotiol, hexadecanotiol e octadecanotiol

- caracterizar defeitos e grau de cobertura em monocamadas auto-arranjadas de octadecanotiol sobre superfícies de ouro

- obter o coeficiente de velocidade de transferência de carga em monocamadas de glutationa sobre ouro

- cinética de adsorção de citocromo c de corração de cavalo sobre monocamadas de carboxitiol em superfícies de ouro

- influência do processo de adsorção de $\beta$-lactoglobulina em aço inoxidável sobre o mecanismo e cinética de corrosão do material metálico

- avaliação do comportamento capacitivo de um compósito polipirrol/poliimida

- estudar os mecanismos de condução iônica, eletrônica e características de troca iônica de filmes de polianilina

- avaliar a cinética de transferência de elétrons em monocamadas auto-organizadas de complexos de inclusão entre $\alpha$-, ou $\beta$-ciclodextrina e brometo de $\mathrm{N}$-(n-octil)-N'-(10-mercaptodecil)-4,4'-bipiridínio 
Por outro lado, devido ao grande interesse na investigação de processos de superfícies em tempo real foi conduzida a primeira aplicação de sistemas SPR por Liedberg e colaboradores em $1983^{85}$. Mediante estudos de adsorção do antígeno $\gamma$-globulina sobre a superfície de um filme de prata e posterior adição do anticorpo $\gamma$-globulina em concentrações variadas, foi possível verificar que o sistema SPR pode ser aplicado de forma extremamente sensível e seletiva. Neste sentido, tem havido um crescente interesse em estudos de interação específica entre biomoléculas, com o propósito de investigar a afinidade e a cinética de interação intermolecular.

A análise por interação bioespecífica em tempo real através de SPR tem adquirido crescente interesse devido ao conjunto de informações que podem fornecer acerca das propriedades de biomoléculas, como modelos de interação, afinidade intermolecular e constantes cinéticas $^{86}$. Com o propósito de investigar propriedades ópticas por sistema SPR duas configurações têm sido aplicadas para o estudo da interface: (1) detecção por interação biomolecular direta sobre a superfície do metal e (2) detecção por interação em matriz hidrogel. Para a investigação uma das moléculas é ligada à superfície do sensor ou à matriz, de forma que as interações com outras moléculas promovam deslocamentos no ângulo de ressonância SPR, que está diretamente relacionado com a quantidade de moléculas que interagem com a superfície do sensor.

$\mathrm{O}$ uso de matriz hidrogel possibilita que uma das moléculas seja covalentemente ligada de forma a proporcionar um maior número de sítios de interação específica por área de superfície, além disto a capacidade de imobilização de biomoléculas sobre a matriz é superior e, nesse sentido, o uso de uma matriz promove um aumento na sensibilidade do sistema ${ }^{87}$ e melhora a acessibilidade das molécu$\operatorname{las}^{88}$. Desta forma, o uso de SPR possibilita a análise por interação biomolecular em tempo real sem o uso de reagentes marcadores, de forma que tal sistema pode ser usado para determinar a concentração de moléculas, afinidade e cinética de interação ${ }^{89}$, bem como se expande aos mais variados campos de atividade. A Tabela 3 apresenta algumas aplicações do SPR relacionadas ao desenvolvimento de dispositivos sensores.
As potencialidades analíticas da espectroscopia de impedância eletroquímica, ressonância de plásmons de superfície e microbalança de cristal de quartzo, bem como a importância de suas aplicações às investigações e caracterizações de processos superficiais e interfaciais estão intimamente ligadas ao desenvolvimento de métodos confiáveis. Neste contexto, o emprego combinado destas técnicas tem sido explorado com o propósito de fornecer informações complementares que possibilitem interpretações seguras sobre o sistema avaliado (Esquema 1).

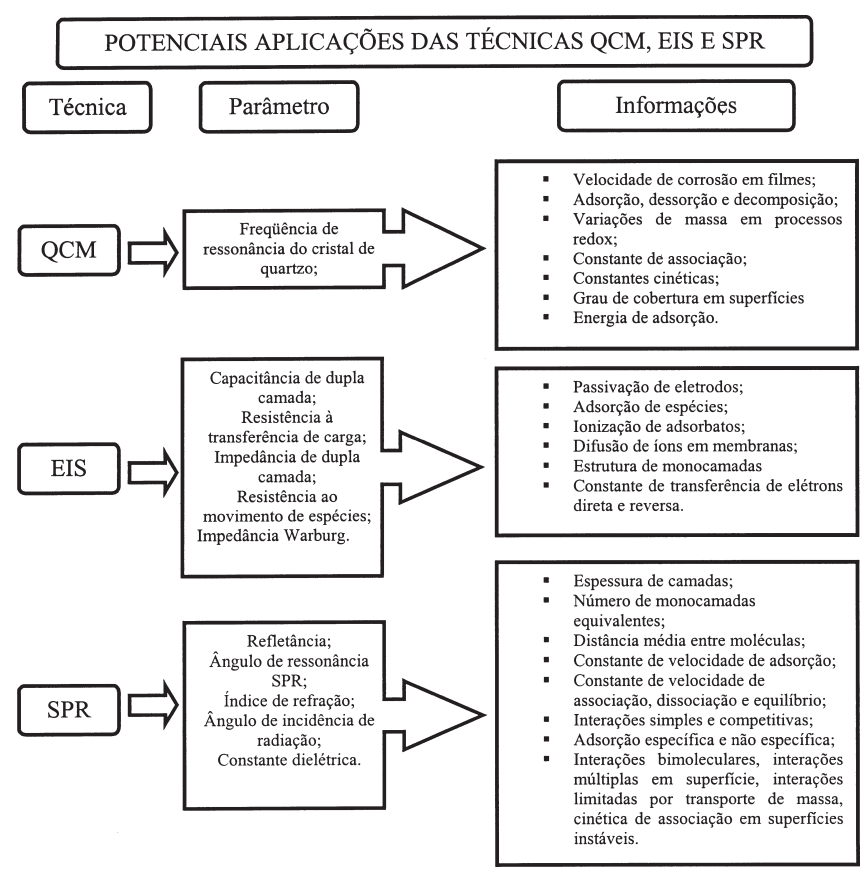

Esquema 1. Principais parâmetros e informações obtidos em aplicações de QCM, EIS e SPR

Tabela 3. Exemplos de aplicação da ressonância de plásmons de superfície na investigação de processos interfaciais e superficiais

\begin{tabular}{|c|c|c|}
\hline Aplicação & Propósito & Ref. \\
\hline Filmes orgânicos & $\begin{array}{l}\text { - avaliar a espessura, permissividade e esturtura de filmes de azobenzeno } \\
\text { - estudar a espessura, constante dielétrica e condutividade de filmes de } \\
\text { polipirrol modificados com } 3(\omega \text {-mercaptoundecil })\end{array}$ & 117 \\
\hline Filmes de Langmuir-Blodgett & $\begin{array}{l}\text { - calcular a espessura, índice de refração e coeficiente de extinção } \\
\text { de filmes de calix-4resorcinareno }\end{array}$ & 118 \\
\hline Monocamadas auto-organizadas & $\begin{array}{l}\text { - estudar o grau de cobertura, espessura de filmes de DNA modificado com } \\
\text { mercapto-hexanol, bem como cinética de adsorção e desorção } \\
\text { - avaliar a cinética de formação de monocamadas auto-arranjadas } \\
\text { de poli( } \gamma \text {-benzil L-glutamato) sobre ouro } \\
\text { - estudar a interação de monocamadas de ácido ditiobis(4-butirilamino- } \\
\text { m-fenilborônico) com frutose, manose e galactose }\end{array}$ & 120 \\
\hline Filmes protéicos & $\begin{array}{l}\text { - estudar a interação de proteínas ( } \sigma \text {-endotoxinas) da bactéria Bacillus } \\
\text { thuringiensis, constantes de velocidade para associação, dissociação e } \\
\text { constante de afinidade }\end{array}$ & 122 \\
\hline
\end{tabular}


Com este propósito, Vogel e colaboradores ${ }^{90}$ empregaram simultaneamente a espectroscopia de impedância e a ressonância de plásmons de superfície na investigação de interações entre a toxina do cólera e "monosialogangliosídeos". Para gerar informações quantitativas para lipídeos em suporte sólido foram conduzidas medidas de SPR. A EIS foi empregada para possibilitar o cálculo do conteúdo de receptor na camada lipídica. Offennhausser e colaboradores ${ }^{91}$ investigaram propriedades de monocamadas auto-organizadas de alcanotiois e tiolipídeos sobre ouro. Empregando SPR e EIS foram avaliadas a espessura média da monocamada e a cinética de transferência de elétrons e de adsorção. A QCM e EIS foram, recentemente, aplicadas em conjunto por Guiseppe-Elie e colaboradores ${ }^{81}$ para investigações de hibridização de DNA, mediante avaliação da impedância e freqüência associadas às mudanças na conformação do oligonucleotídeo.

Com o propósito de reduzir as interferências provenientes da associação entre a QCM e a EIS, Yao e colaboradores ${ }^{92}$ desenvolveram uma nova estratégia para aplicação simultânea de cristais piezelétricos de quartzo e impedância eletroquímica (impedância em cristal piezelétrico de quartzo - ICPQ). Este sistema híbrido foi aplicado na investigação da adsorção de albumina bovina sobre eletrodos de ouro e platina, uma vez que a ICPQ possibilita investigações de massa de eletrodos, viscoelasticidade de filme, viscosidade/densidade local de solução próximo à superfície do eletrodo e capacitância interfacial. A Tabela 4 apresenta algumas aplicações simultâneas das técnicas, tendo como enfoque suas potencialidades individuais.

\section{CONCLUSÕES E PERSPECTIVAS FUTURAS}

Este trabalho ilustra algumas aplicações potenciais da espectroscopia de impedância eletroquímica, ressonância de plásmons de superfície e microbalança de cristal de quartzo tendo como enfoque os fenômenos interfaciais e superficiais e, como propósito, apresentar o auxílio que estas técnicas podem fornecer no procedimento de construção de dispositivos sensores. A importância do uso combinado destas técnicas tem por fundamento as capacidades investigativas atribuídas a cada uma. A QCM e a SPR são capazes de fornecer medidas de dinâmica de eventos em tempo real, sendo que a QCM pode proporcionar informações sobre as variações na massa do sistema, conduzindo a importantes informações sobre as transferências de espécies em filmes. A SPR pode complementar estas medidas mediante informações sobre a dinâmica dos processos com alto nível de sensibilidade, e a EIS pode gerar informações sobre as propriedades condutoras dos sistemas desenvolvidos.

Desta forma, a aplicação conjunta da EIS, SPR e QCM constitui uma poderosa ferramenta no estudo de um grande número de propriedades relacionadas aos processos interfaciais e superficiais, de forma a implicar maior confiabilidade às investigações destes processos. Estas técnicas apresentam-se como ferramentas promissoras para aplicação, tanto em configurações individuais como de forma conjunta ou mesmo simultânea, in situ dos sistemas investigados. Conseqüientemente, suas aplicações ao desenvolvimento otimizado de filmes para aplicação como suportes de/ou receptores em sistemas

Tabela 4. Exemplos da aplicação combinada das técnicas SPR, QCM e EIS na investigação de processos interfaciais e superficiais

Aplicação Propósito Ref.

EIS e QCM

SPR e EIS

QCM e SPR

SPR, QCM e EIS
- uso da EIS no estudo da relação entre o movimento de íons e moléculas polarizáveis com a hibridização do DNA e aplicação da QCM para distinção entre processos de hibridização complementar e não-complementar

- monitoramento em tempo-real através da QCM da adsorção de protamina seguida pela adsorção de heparina, bem como otimização dos processos de interação (protamina-heparina) e avaliação dos efeitos da viscosidade e densidade da solução de heparina no sinal analítico. A EIS foi aplicada para avaliação dos parâmetros físicos do sistema: resistência da solução, resistência à transferência eletrônica, capacitância da dupla camada elétrica e suas relações com as concentrações de heparina

- aplicação da EIS na avaliação do comportamento capacitivo de filmes de polianalina modificados com trissulfeto de molibdênio e uso da QCM para monitoramento do transporte de massa no compósito, para elaboração de um mecanismo de transporte de cátions, ânions e espécies neutras no filme

- aplicação da SPR no monitoramento em tempo-real da formação de multicamadas de avidina-biotina sobre ouro e estimativa da concentração de moléculas em superfície e investigação, por EIS, da relação entre o constituinte da camada e a resistência à transferência de carga e capacitância de dupla camada

- estudo da adsorção e dessorção de espécies sobre materiais condutores e suas influências na transferência interfacial de elétrons e uso da SPR no monitoramento em tempo-real da formação das bicamadas lipídicas, e estimativa de densidade de lipídeos em superfície

- uso da SPR para avaliação da cinética de adsorção de proteínas modificadas com endoglucanase e aplicação da QCM para avaliação da rigidez da camada protéica adsorvida

- SPR aplicada na obtenção da espessura do filme durante o processo de crescimento, fornecendo informações sobre a dinâmica de formação das monocamadas; as informações da QCM possibilitaram uma diferenciação entre adsorção e fusão das vesículas de diftanoilfosfatidilcolina sobre as monocamadas auto-organizadas, enquanto a EIS possibilitou um monitoramento dos parâmetros físicos dos filmes 
sensores são fundamentais, pois possibilitam um conhecimento detalhado de propriedades importantes dos sistemas investigados.

Como perspectiva futura verifica-se um grande desenvolvimento e aplicação da QCM e SPR combinadas às técnicas eletroquímicas (fundamentalmente à EIS), de forma a gerar um conjunto grande e informativo de dados, simultaneamente conduzindo a medidas detalhadas dos sistemas interfaciais e superficiais e, assim, contribuindo de maneira decisiva para o entendimento destes processos, bem como para o fortalecimento e a expansão das aplicações das microbalanças de cristal de quartzo eletroquímica e ressonância de plásmons de superfície eletroquímica.

\section{REFERÊNCIAS}

1. Valcárcel, M.; Rios, A.; Anal. Chim. Acta 1999, 400, 425.

2. Zhang, S.; Wright, G.; Yang, Y.; Biosens. Bioelectron. 2000, 15, 273.

3. Thévenot, D. R.; Toth, K.; Durst, R. A.; Wilson, G. S.; Biosens. Bioelectron. 2001, 16, 121 .

4. Fraser, D.; Biosensors in the Body: continuous in vivo monitoring, Wiley: New York, 1997

5. Jacobson, G. A.; Winkeler, M.; Mater. Performance 2000, 39, 120.

6. Swalen, J. D.; Allara D. L.; Andrade, J. D.; Chandross, E. A.; Garoff, S.; Israelachvili, J.; McCarthy, T. J.; Murray, R.; Pease, R. F.; Rabolt, J. F.; Wynne, K. J.; Yu, H.; Langmuir 1987, 3, 932.

7. Pereira, A. C.; Santos, A. S.; Kubota, L. T.; Quim. Nova 2002, 25, 1012.

8. Damos, F. S.; Sotomayor, M. D. T.; Kubota, L. T.; Tanaka, S. M. C. N.; Tanaka, A. A.; Analyst 2003, 128, 255.

9. Freire, R. S.; Pessoa, C. A.; Mello, L. D.; Kubota, L. T.; J. Braz. Chem. Soc. 2003, 14, 230.

10. Freire, R. S.; Kubota, L. T.; Analyst 2002, 127, 1502.

11. Carvalho, R. M. de; Neto, G. D.; Kubota, L. T.; Anal. Lett. 2000, 33, 425.

12. Santos, A. D.; Gorton, L.; Kubota, L. T.; Electrochim. Acta 2002, 47, 3351.

13. Fujiwara, H.; Toyoshima, Y.; Kondo, M.; Matsuda, A.; Sol. Energy Mater. Sol. Cells 2001, 66, 209.

14. Kim, S. H.; Choi, S. W.; Suh, H. J.; Jin, S. H.; Gal, Y. S.; Koh, K.; Dyes Pigm. 2002, 55, 17.

15. Geurtz, J.; Surf. Sci. Rep. 1993, 18, 1.

16. Schweiss, R.; Werner, C.; Knoll, W.; J. Electroanal. Chem. 2003, 540, 145.

17. Lieber, C. M.; Liu, J.; Inorg. Chim. Acta 1996, 243, 305.

18. Stoica, T. F.; Teodorescu, V. S.; Blanchin, M. G.; Stoica, T. A.; Gertner, M.; Losendo, M.; Zaharescu, M.; Mater. Sci. Eng., B 2003, 101, 222

19. Pavey, K. D.; Hunter, A. C.; Paul, F.; Biosens. Bioelectron. 2003, 18, 1349.

20. Striebel, C.; Brecht, A.; Gauglitz, G.; Biosens. Bioelectron. 1994, 9, 139.

21. Ratner, B. D.; Cardiovasc. Pathol. 1993, 2, S87.

22. Kosslinger, C.; Uttenthaler, E.; Drost, S.; Aberl, F.; Wolf, H.; Brink, G.; Stanglmaier, A.; Sackmann, E.; Sens. Actuators, B 1995, 24, 107.

23. Jiang, J.; Kucernak, A.; J. Electroanal. Chem. 2002, 520, 64

24. Green, R. J.; Frazier, R. A.; Shakesheff, K. M.; Davies, M. C.; Roberts, C. J.; Tendler, S. J. B.; Biomaterials 2000, 21, 1823.

25. O’Sullivan, C. K.; Gilbault, G. G.; Biosens. Bioelectron. 1999, 14, 663.

26. Sauerbrey, G.; Z. Phys. 1958, 155, 206.

27. King, W. H.; Anal. Chem. 1964, 36, 1735.

28. Kanazawa, K. K.; Gordon, J. G.; Anal. Chem. 1985, 57, 1770.

29. MacCallum, J. J.; Alder, J. F.; Analyst 1983, 108, 1169.

30. Alder, J. F.; Drew, P. K. P.; Fielden, P. R. A.; J. Chromatogr., A 1981, 212, 1981.

31. Konash, P. L.; Bastiaans, G. J.; Anal. Chem. 1980, 52, 1929.

32. Nomura, T.; Okuhara, M.; Anal. Chim. Acta 1982, 142, 281.

33. Schumacher, R.; Borges, G.; Kanazawa, K. K.; Surf. Sci. 1985, 163, L621.

34. Muramatsu, H.; Dicks, J. M.; Tamiya, E.; Karube, I.; Anal. Chem. 1987, $59,2760$.

35. Kurosawa, S.; Tawara, E.; Kamo, N.; Kobatake, Y.; Anal. Chim. Acta 1990, $230,41$.

36. Yao, S.-Z.; Zhou, T.-A.; Anal. Chim. Acta 1988, 212, 61.

37. Thompson, M.; Arthur, C. L.; Dhaliwal, G. K.; Anal. Chem. 1986, 58, 1206

38. Cavic-Vlasak, B. A.; Rajakovic, L. J. V.; Fresenius J. Anal. Chem. 1992, 343, 339.

39. Rajakovic, L.; Ghaemmaghami, V.; Thompson, M.; Anal. Chim. Acta 1989, 217,111 .

40. Duncan-Hewitt, W. C.; Thompson, M.; Anal. Chem. 1992, 64, 94.

41. Ward, M. D.; Delawski, E. J.; Anal. Chem. 1991, 63, 886

42. Hillier, A. C.; Ward, M. D.; Anal. Chem. 1992, 64, 2539.

43. Sluyters-Rehbech, M.; Pure Appl. Chem. 1994, 66, 1931.
44. Bard, A. J.; Faulkner, L. R.; Electrochemical Methods: Fundamentals and Applications, Wiley: New York, 1980.

45. Zoltowski, P.; J. Electroanal. Chem. 1994, 375, 45.

46. Alves, V. A.; Brett, C. M. A.; Electrochim. Acta 2002, 47, 2081.

47. Sluyters-Rehbach, M.; Sluyters, J. H.; J. Electroanal. Chem. 1970, 26, 237.

48. Homola, J.; Yee, S. S.; Gauglitz, G.; Sens. Actuators, B 1999, 54, 3.

49. Kretschman, E.; Raether, H.; Z. Naturforsch., A: Phys. Sci. 1968, 23, 2135.

50. Homola, J.; Koudela, I.; Yee, S. S.; Sens. Actuators, B 1999, 54, 16.

51. Bunde, R. L; Jarvi, E. J.; Rosentreter, J. J.; Talanta 1998, 46, 1223.

52. Rickert, J.; Weiss, T.; Kraas, W.; Jung, G.; Gopel, W.; Biosens. Bioelectron. 1996, 11, 591.

53. Caruso, F.; Furlong, D. N.; Niikura, K.; Okahata, K.; Colloids Surf., B 1998 10, 199.

54. Palecek, E.; Fojta, M.; Tomschik, M.; Wang, J.; Biosens. Bioelectron. 1998, 13,621

55. Cosnier, S.; Perrot, H.; Wessel, R.; Electroanalysis 2001, 13, 971.

56. Oyama, N.; Kelly, A. J.; J. Phys. Chem. 1991, 95, 9579.

57. Komura, T.; Niu, G. Y.; Yamaguchi, T.; Asano, M.; Electrochim. Acta 2003, 48,631 .

58. Snook, G. A.; Bond, A. M.; Flatcher, S.; J. Electroanal. Chem. 2002, 526 , 1.

59. Mao, Y. A.; Wei, W. Z.; Zhang, J. Z.; Peng, H.; Wu, L.; Microchem. J. 2001, $70,133$.

60. Kim, H. J.; Kwak, S.; Kim, Y. S.; Seo, B. I.; Kim, E. R.; Lee, H.; Thin Solid Films 1998, 191, 327

61. Shen, D. Z.; Wu, X.; Liu, X. Y.; Kang, Q.; Chen, S. H.; Microchem. J. 1999, 63,322

62. Scendo M.; Malyszko, J.; Monatsh. Chem. 1997, 128, 123.

63. Shen, D. Z.; Huang, M. H.; Chow, L. M.; Yang, M. S.; Sens. Actuators, B 2001, 77, 664 .

64. Fey, G. T.-K.; Weng, Z.-X.; Chen, J.-G.; Kumar, T. P.; Mater. Chem. Phys. 2003, 80, 309.

65. Wang, Q.; Li, N.; Talanta 2001, 55, 1219.

66. Antoine, O.; Bultel, Y.; Durand, R.; J. Electroanal. Chem. 2001, 499, 85.

67. Braun, A.; Bartsch, M.; Merlo, O.; Schnyder, B.; Schaffner, B.; Kotz, R.; Haas, O.; Wokaun, A.; Carbon 2003, 41, 759.

68. Salkind, A. J.; Singh, P.; Cannone, A.; Atwater, T.; Wang, X.; Reisner, D.; J. Power Sources 2003, 116, 174.

69. Mirabedini, S. M.; Thompson, G. E.; Morandian, S.; Scantlebury, J. D.; Prog. Org. Coat. 2003, 46, 112.

70. Angelini, E.; Grassini, S.; Rosalbino, F.; Fracassi, F.; d'Agostino, R.; Prog. Org. Coat. 2003, 46, 107.

71. Silva, L. M. da; Faria, L. A. de; Boodts, J. F. C.; J. Electroanal. Chem. 2002, 532, 141.

72. Cheng, T.-J.; Lin, T.-M.; Chang, H.-C.; Anal. Chim. Acta 2002, 462, 261.

73. Rickert, J.; Gopel, W.; Beck, W.; Jung, G.; Heiduschka, P.; Biosens. Bioelectron. 1996, 11, 757.

74. Farace, G.; Lillie, G.; Hianik, T.; Payne, P.; Vadgama, P.; Bioelectrochemistry 2002, 55, 1 .

75. Ouerghi, O.; Senillou, A.; Jaffrezic-Renault, N.; Martelet, C.; Ben Ouada, H. J.; Cosnier, S.; Electroanal. Chem. 2001, 501, 62.

76. Sakly, N.; Souiri, M.; Romdhane, F. F.; Bem Ouada, H.; Jaffrezic-Renault, N.; Mater. Sci. Eng., C 2002, 21, 47.

77. Cui, X. Q.; Pei, R. J.; Wang, X. Z.; Yang, F.; Ma, Y.; Dong, S. J.; Yang, X. R.; Biosens. Bioelectron. 2003, 18, 59 .

78. Diao, P.; Guo, M.; Tong, R. T.; J. Electroanal Chem. 2001, 495, 98.

79. Lillie, G.; Payne, P.; Vadgama, P.; Sens. Actuators, B 2001, 78, 249.

80. Kasen, K. K.; Mater. Sci. Eng., B 2001, 83, 97.

81. Guiseppi-Elie, A.; Gheorghe, M.; Biosens. Bioelectron. 2004, 19, 95.

82. Strasak, L.; Dvorak, J.; Hason, S.; Vetterl, V.; Bioelectrochemistry 2002, 56,37 .

83. Munichandraiah, N.; Prasad, K. R.; Synth. Met. 2002, 126, 62.

84. Kim, Y.; Teshima, K.; Kobayashi, N.; Electrochim. Acta 2000, 45, 1549.

85. Liedberg, B.; Nylander, C.; Lundstrom, I.; Sens. Actuators, B 1983, 4, 299.

86. Lundstron, I.; Biosens. Bioelectron. 1994, 9, 725.

87. Liedberg, B.; Lundstrom, I.; Stenberg, E.; Sens. Actuators, B 1993, 11, 63.

88. Lofas, S.; Malmqvist, M.; Ronnberg, I.; Stenberg, E.; Liedberg, B.; Lundstrom, I.; Sens. Actuators, B 1991, 5, 79.

89. Jonsson, U.; Malmqvist, M.; Adv. Biosens. 1992, 2, 291.

90. Terrettaz, S.; Stora, T.; Duschl, C.; Vogel, H.; Langmuir 1993, 9, 1361.

91. Lingler, S.; Rubinstein, I.; Knoll, W.; Offenhaussen, A.; Langmuir 1997, 13, 7085 .

92. Luo, S. L.; Chen, J. H.; Kuang, Y. F.; Zhou, H. H.; Yao, S. Z.; Thin Solids Films 2003, 424, 208.

93. Kubono, A.; Yuasa, N.; Shao, H.-L.; Umemoto, S.; Okui, N.; Appl. Surf. Sci. 2002, 193, 195. 
94. Stevenson, K.; Miyashita, N.; Smieja, J.; Mazur, U.; Ultramicroscopy 2003 , 97, 271.

95. Stalgren, J. J. R.; Claesson, P. M.; Warnheim, T.; Adv. Colloid Interfaces Sci. 2001, 89, 383.

96. Miura, Y.; Sasao, Y.; Dohi, H.; Nishida, Y.; Kobayashi, K.; Anal. Biochem. 2002, 310, 27.

97. Qu, D.; Morin, M.; J. Electroanal. Chem. 2002, 524, 77.

98. Marxer, C. G.; Coen, M. C.; Schlapbach, L.; J. Colloid Interface Sci. 2003, 261,291

99. Mao, Y.; Wei, W.; He, D.; Nie, L.; Yao, S.; Anal. Biochem. 2002, 306, 23.

100. Syritski, V.; Opik, A.; Forsen, O.; Electrochim. Acta 2003, 48, 1409.

101. Feher, K.; Inzelt, G.; Electrochim. Acta 2002, 47, 3551.

102. Kanungo, M.; Kumar, A.; Contractor, A. Q.; J. Electroanal. Chem. 2002, $528,46$.

103. Gou, W.; Wang, J.; Wang, C.; He, J.-Q.; He, X.-W.; Cheng, J.-P.; Tetrahedron Lett. 2002, 43, 5665.

104. Wang, C.; Chen, F.; He, X.-W.; Anal. Chim. Acta 2002, 464, 57.

105. Schweiss, R.; Werner, C.; Knoll, W.; J. Electroanal. Chem. 2003, 540, 145.

106. Miyashita, T.; Aoki, A.; Abe, Y.; Mol. Cryst. Liq. Cryst. Sci. Technol. 1999, 327, 77.

107. Saliba, R.; Ravaine, S.; Mingotaude, C.; Agricole, B.; J. Phys. Chem. B 1999, 103, 9712.

108. Subramanian, R.; Lakshminarayanan, V.; Electrochim. Acta 2000, 45, 4501

109. Diao, P.; Guo, M.; Tong, R. T.; J. Electroanal. Chem. 2001, 495, 98.

110. Zhou, A.; Xie, Q.; Wu, Y.; Cai, Y.; Nie, L.; Yao, S.; J. Colloid Interface Sci. 2000, 229, 12.

111. Nahir, T. M.; Bowden, E. F.; Langmuir 2002, 18, 5283.

112. Omanovic, S.; Roscoe, S. G.; J. Colloid Interface Sci. 2000, 227, 452.

113. Iroh, J. O.; Levine, K.; J. Power Sources 2003, 117, 267.
114. Mori, K.; Yamaguchi, T.; Takahashim, K.; Komura, T.; Bull. Chem. Soc. Jpn. 2000, 73, 19.

115. Yan, J. C.; Dong, S. J.; Li, J. H.; Chen, W. Q.; J. Electrochem. Soc. 1997, $144,3858$.

116. Zhou, M.; Otomo, A.; Yokoyama, S.; Mashiko, S.; Thin Solid Films 2001, $393,114$.

117. Georgiadis, R.; Peterlinz, K. P.; Peterson, A. W.; Rahn, J. R.; Grassi, J. H.; Langmuir 2000, 16, 6759.

118. Hassan, A. K.; Nabok, A. V.; Ray, A. K.; Lucke, A.; Smith, K.; Stirlimg, C. J. M.; Davis, F.; Mater. Sci. Eng., C 1999, 8, 251.

119. Georgiadis, R.; Peterlinz, K. P.; Peterson, A. W.; J. Am. Chem. Soc. 2000, $122,3166$.

120. Willians, A. J.; Gupta, V. K.; Thin Solids Films 2003, 423, 228.

121. Lee, M.; Kim, T.-I.; Kim, K.-H.; Kim, J.-H.; Choi, M.-S.; Choi, H.-J.; Koh, K.; Anal. Biochem. 2002, 310, 163.

122. Okumura, S.; Akao, T.; Mizuki, E.; Ohba, M.; Inouye, K.; J. Biochem. Biophys. Methods 2001, 47, 177.

123. Kang, X.; Jin, Y.; Cheng, G.; Dong, S.; Langmuir 2002, 18, 1713.

124. Nabok, A. V.; Hassan, A. K.; Ray, A. K.; Omar, O.; Kalchenko, V. I.; Sens. Actuators, B 1997, 45, 115.

125. Hassan, A. K.; Ray, A. K.; Nabok, A. V.; Davis, F.; Sens. Actuators, B 2001, 77, 638.

126. Fusalba, F.; Belanger, D.; J. Mater. Res. 1999, 14, 1805.

127. Yang, F.; Cui, X.; Yang, X.; Biophys. Chem. 2002, 99, 99.

128. Linder, M.; Szilvay, G. R.; Nakari-Setala, T.; Soderlund, H.; Penttila, M.; Protein Sci. $2002,11,2257$.

129. Naumann, R.; Schiller, S. M.; Geiss, F.; Grohe, B.; Hartman, K. B.; Karcher, I.; Koper, I.; Lubben, J.; Vasilev, K.; Knoll, W.; Langmuir 2003, 19,5435 . 